\title{
Evaluation of Upper Endoscopic and Endoscopic Ultrasound Features in the Differential Diagnosis of Gastrointestinal Stromal Tumors and Leiomyomas in the Upper Gastrointestinal Tract
}

\author{
Rolandas Vaicekauskas $^{a} \quad J_{\text {Jrgita Urboniené }}{ }^{b} \quad J_{\text {Juozas Stanaitis }}^{a}$ \\ Jonas Valantinas ${ }^{\text {a }}$ \\ ${ }^{a}$ Clinic of Gastroenterology, Nephrourology, and Surgery, Institute of Clinical Medicine, Faculty of Medicine, \\ Vilnius University, Vilnius, Lithuania; ${ }^{b}$ Vilnius University Hospital Santaros Klinikos, Vilnius, Lithuania
}

\section{Keywords}

Gastrointestinal stromal tumor · Leiomyoma - Upper endoscopy · Endoscopic ultrasound

\begin{abstract}
Background: Differentiation between benign and malignant subepithelial lesions (SELs) in the upper gastrointestinal tract (UGT) leads to far-reaching clinical consequences. An accurate diagnosis can be challenging because of the insufficient diagnostic yield of upper endoscopy, endoscopic ultrasound (EUS), and different types of biopsy. Aim: Our aim was to reveal the efficacy of upper endoscopic and EUS features for the differential diagnosis of hypoechogenic SELs (gastrointestinal stromal tumors [GISTs] and leiomyomas) in the UGT. Materials and Methods: The research covers a case series study of 27 hypoechogenic SELs in the UGT between 2012 and 2015 at Vilnius University Hospital Santaros Klinikos. Upper endoscopic and EUS features of SELs were recorded. In order to standardize the diagnostic approach to GISTs and leiomyomas, we assigned scores for seven upper endoscopic and EUS features. Results: The mean total scores in the GIST group were significantly higher than those in the leiomyoma group: $3.25 \pm 1.71$ and $0.53 \pm 0.83(p<0.0001)$, respectively. Increment by one score increased the odds ratio for GIST 5.87 times (95\% Cl 1.63-21.11; $p=0.007$ ). The total score demonstrated very good discriminatory features of GISTs against leiomyomas (area under the receiver operat-
\end{abstract}

ing characteristic curve 0.94 [0.86-1]). The cutoff value of 1.5 total score indicated $83.3 \%$ sensitivity and $93.3 \%$ specificity in diagnosing GISTs. Conclusions: Upper endoscopy and EUS are useful methods in making a definite diagnosis of SELs. Their diagnostic accuracy for the differential diagnosis of GISTs and leiomyomas is sufficient. @ 2019 S. Karger AG, Basel

\section{Introduction}

The term upper gastrointestinal tract (UGT) subepithelial lesions (SELs) is applied to a mass or a bulge covered by normal-appearing mucosa identified during upper endoscopy. These SELs are usually found incidentally. The incidence of gastric SELs was reported to be $0.36 \%$ [1]. According to our data the prevalence of SELs in the UGT was $1.37 \%$, and most of them (73\%) were diagnosed in the stomach [2]. Differentiation between benign and malignant lesions is of great clinical importance. The accurate diagnosis can be challenging because of the insufficient diagnostic yield of the upper endoscopy, endoscopic ultrasound (EUS), and different types of biopsy. The management depends on the diagnosis determined by using various diagnostic methods. Gastrointestinal stromal tumors (GISTs) are the most common mesenchymal neoplasms in the gastrointestinal tract and are most regularly found in the stomach. The risk of progres- 


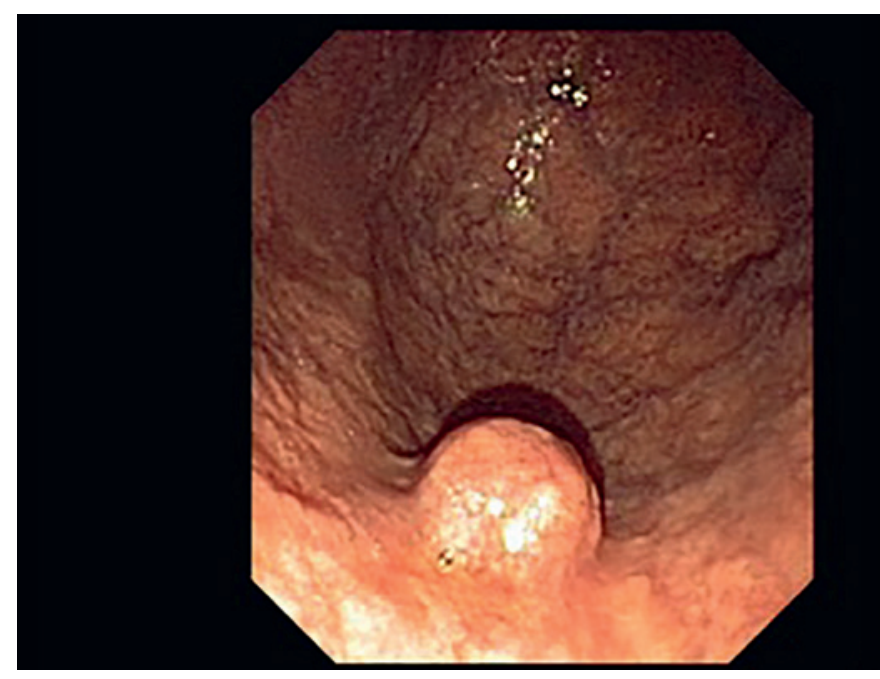

Fig. 1. Upper endoscopic image of a stomach gastrointestinal stromal tumor.

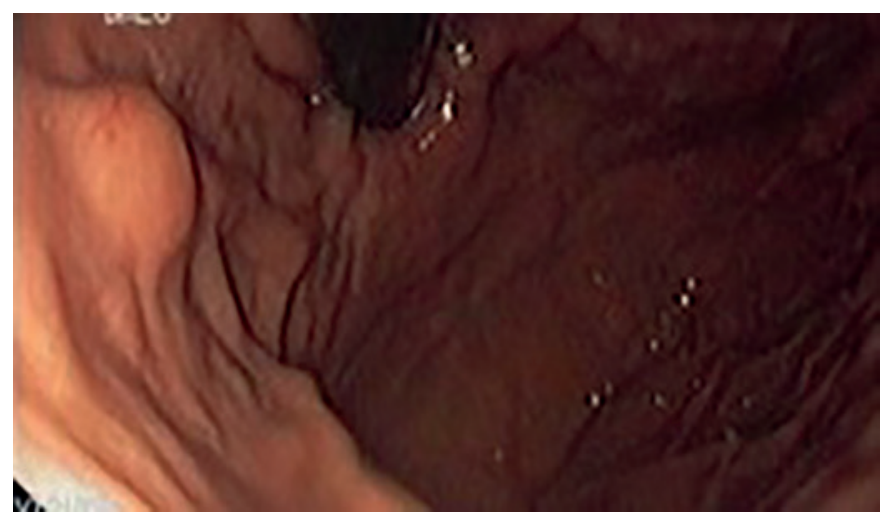

Fig. 2. Upper endoscopic image of a stomach leiomyoma.

sion and metastases depends on the GIST's size, location, and mitotic rate. According to Miettinen et al. [3] the malignancy risk of GISTs can be nonexistent, very low, low, moderate, or high. Contrary to GISTs, leiomyomas are benign SELs and are mostly located in the esophagus. However, a few cases of GISTs have been found in the esophagus and leiomyomas in the stomach [4-6]. For the establishment of a more accurate diagnosis, it is vital to apply an appropriate treatment or a follow-up subsequently. Thus, it is not enough to perform EUS only [7]. According to this study, in $42.9 \%$ of cases in whom preoperative EUS was performed but without histological examination after surgery, they appeared to be benign. It seems that preoperative biopsy of SELs should be performed. However, there is no single management algorithm of these lesions. Could we increase the accuracy of EUS in the diagnosis of SELs? In our study we aimed to evaluate upper endoscopic and EUS features in the differential diagnosis of GISTs and leiomyomas in the UGT.

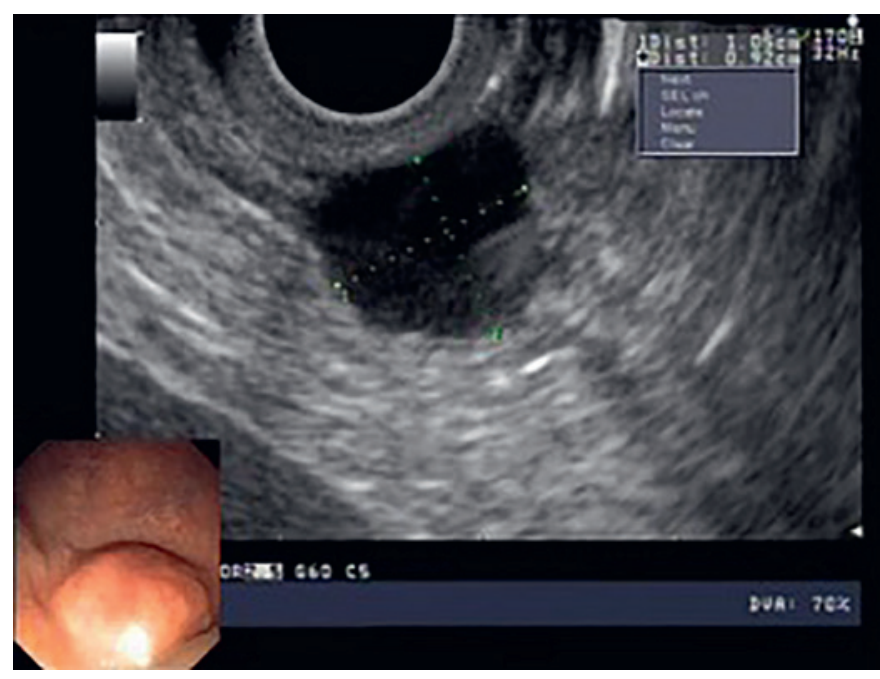

Fig. 3. Endoscopic ultrasound image of a stomach gastrointestinal stromal tumor.

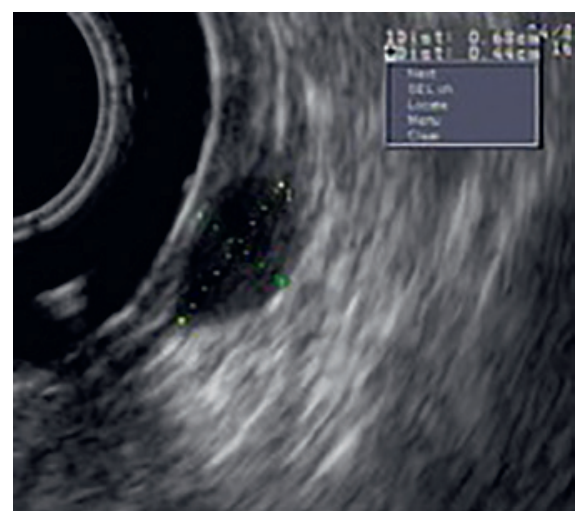

Fig. 4. Endoscopic ultrasound image of a stomach leiomyoma.

We propose a scoring system with a cutoff value to predict GIST probability. Indeed, these are only initial retrospective results with some limitations. In view of advanced EUS imaging techniques, SEL size and different types of SELs, our proposed scoring system should be modified and evaluated in future prospective studies.

\section{Materials and Methods}

A case series study of 27 hypoechogenic SELs in the UGT was undertaken between 2012 and 2015 at Vilnius University Hospital Santaros Klinikos. Twelve GISTs and 15 leiomyomas were histologically confirmed after deep biopsy via the endoscopic submucosal dissection technique [8]. Subjects were enrolled into the study if they met the following inclusion criteria: (a) presence of UGT solid SELs, (b) presence of SELs with an endophytic or exophytic (meagerly prominent) growth pattern, (c) patient's age between 18 and 80 years, (d) no coagulation abnormalities (prothrombin $>50 \%$ and platelet count $>50,000$ ), no use of anticoagulants, and (e) patient's written consent. Exclusion criteria 
were (a) refusal to provide informed consent, (b) pregnancy, and (c) severe comorbidities.

Upper endoscopy and EUS were performed on an inpatient and outpatient basis with intravenous sedation by one endoscopist before histopathological confirmation of the SELs. Upper endoscopy was done with Olympus endoscopes (Olympus Evis Exera II GIF-Q165 and GIF-H180). Lesion location, size, grown pattern, appearance of the overlaying mucosa, convergence of the folds towards the lesion (Schindler's sign), and consistence were evaluated during the upper endoscopy examination (Fig. 1,2). EUS was done with radial and linear instruments with a distal water-filled balloon (Olympus GF type UE 160-AL5 and GF type 140P-AL5). Size, shape, borders, heterogeneity, echogenicity, layer of origin, marginal halo sign, cystic changes, and calcifications were recorded (Fig. 3, 4). SEL size was measured in millimeters. Lesions with equal vertical and horizontal measurements or the vertical measure exceeding $3 / 4$ of the horizontal one were considered if they had a round shape. In other cases, the lesion shape was considered to be oval or other type. The echogenicity of the lesion was established visually comparing it with the surrounding muscularis propria layer (IV). If the lesion was slightly "brighter" than the surrounding muscle layer, we termed it hypoechogenic hyperechogenic. If the lesion's echogenicity was equal to the IVth layer, we termed it hypoechogenic isoechogenic. If the lesion was slightly "darker" than the IVth layer, we termed it hypoechogenic hypoechogenic. The lesion with the marginal halo sign had a hypoechogenic ring around its peripheral part and a slightly hypoechogenic hyperechogenic central part. In order to standardize the diagnostic approach to GISTs and leiomyomas, we assigned scores for seven upper endoscopic and EUS features. Scores are provided in Table 1.

\section{Statistical Analysis}

Statistical calculations were performed using SPSS version 20.0 for Windows (SPSS Inc., Chicago, IL, USA). Descriptive statistics for categorical and discrete variables are presented by absolute and relative frequencies, and for continuous variables by mean values, standard deviation, and ranges. Differences in sex and EUS findings between leiomyomas and GISTs were assessed using the $\chi^{2}$ test or Fisher's exact test. Differences between the means of the continuous variables were tested using the Mann-Whitney test. The receiver operating characteristic curve was constructed to examine the diagnostic test performance, and the area under the receiver operating characteristic curve (AUC) was assessed. The Youden index was computed to estimate variables' cutoff values. In order to assess the impact of independent variables on the dependent variables, single and multiple regression models were produced. A $p$ value $<0.05$ was considered statistically significant.

$G^{*}$ Power post hoc sample size analysis revealed that the sample size of 27 was adequate $(\alpha=0.05, \beta=0.87$, effect size $=0.6)$. The null hypothesis was that EUS was accurate for diagnosing GIST in $50 \%$ of cases and the alternative hypothesis was that EUS could accurately differentiate GIST in $80 \%$ of cases.

\section{Results}

The data of 27 patients with a histologically confirmed GIST or leiomyoma diagnosis were analyzed. Patients' median age was $58.67 \pm 12.47$ years (range $26-82$ ). Patients with GISTs were older by 11.10 years $(p=0.014)$; $74.1 \%$ of patients were females. The rates of GISTs and
Table 1. Proposed diagnostic scoring table of upper endoscopic and EUS features in the prediction of GISTs

\begin{tabular}{ll}
\hline Parameter & Score \\
\hline Localization & \\
$\quad$ Esophagus, stomach (cardia) & 0 \\
$\quad$ Stomach (other parts) and duodenum & 1 \\
Schindler's sign & \\
$\quad$ No & 0 \\
$\quad$ Yes & 1 \\
Shape of the lesion & 0 \\
$\quad$ Oval or other & 1 \\
$\quad$ Round & \\
EUS heterogeneity & 0 \\
$\quad$ Homogeneous & 1 \\
$\quad$ Heterogeneous & 0 \\
EUS echogenicity (compared to $4^{\text {th }}$-layer echogenicity) & \\
$\quad$ Hypoechogenic hypoechogenic and isoechogenic & 1 \\
Hypoechogenic hyperechogenic & \\
EUS “halo" sign & 0 \\
$\quad$ No & 1 \\
$\quad$ Yes & \\
EUS cystic changes (anechoic spaces) & 0 \\
$\quad$ No & 1 \\
$\quad$ Yes &
\end{tabular}

EUS, endoscopic ultrasound; GISTs, gastrointestinal stromal tumors.

Table 2. Clinical features of patients with GISTs and leiomyomas

\begin{tabular}{|c|c|c|c|}
\hline Variables & GISTs & Leiomyomas & $p$ value \\
\hline Age, years & $64.83 \pm 9.52$ & $53.73 \pm 12.60$ & 0.014 \\
\hline \multicolumn{4}{|l|}{ Sex } \\
\hline Male & $2(16.7 \%)$ & $5(33.3 \%)$ & 0.326 \\
\hline Female & $10(83.3 \%)$ & $10(66.7 \%)$ & \\
\hline \multicolumn{4}{|c|}{ Clinical symptoms } \\
\hline Absent & $2(16.7 \%)$ & $7(46.7 \%)$ & 0.217 \\
\hline Present & $10(83.3 \%)$ & $8(53.3 \%)$ & \\
\hline
\end{tabular}

GISTs, gastrointestinal stromal tumors.

leiomyomas did not differ between males and females. Symptoms occurred in $83.3 \%$ of GISTs and in $53.3 \%$ of leiomyomas, and in the vast majority of cases it was dyspepsia. Only in 2 cases did leiomyomas cause dysphagia. Asymptomatic GISTs and leiomyomas accounted for $33.3 \%$ of all cases (Table 2).

The location of GISTs and leiomyomas varied; $83.3 \%$ of GISTs were located in the stomach (mostly involving the stomach body, followed by the subcardia and the antrum), while $53.3 \%$ of leiomyomas were located in esophagus and $46.7 \%$ of leiomyomas were located in the stomach (most frequently affecting the cardia and the subcardia of the stomach). The mean size of the SELs was 23.44 $\pm 11.83 \mathrm{~mm}$ (range 7-50). 
Table 3. Upper endoscopic features of patients with GISTs and leiomyomas

\begin{tabular}{|c|c|c|c|}
\hline Variables & GISTs & Leiomyomas & $p$ value \\
\hline Location & & & 0.006 \\
\hline Esophagus & 0 & $8(53.3 \%)$ & \\
\hline Stomach & $10(83.3 \%)$ & $7(46.7 \%)$ & \\
\hline Duodenum & $2(16.7 \%)$ & 0 & \\
\hline \multicolumn{4}{|l|}{ Stomach lesion localization } \\
\hline Cardia & 0 & $3(42.9 \%)$ & 0.288 \\
\hline Subcardia & $3(30.0 \%)$ & $2(28.6 \%)$ & \\
\hline Fundus & $1(10.0 \%)$ & 0 & \\
\hline Body & $4(40.0 \%)$ & $1(14.3 \%)$ & \\
\hline Pylorus & $2(20.0 \%)$ & $1(14.3 \%)$ & \\
\hline Mean lesion size, mm & $26.25 \pm 12.27$ & $19.46 \pm 10.78$ & 0.110 \\
\hline Endoscopic size groups & & & 0.648 \\
\hline$<10 \mathrm{~mm}$ & $1(8.3 \%)$ & $3(23.1 \%)$ & \\
\hline $10-19 \mathrm{~mm}$ & $3(25.0 \%)$ & $4(30.8 \%)$ & \\
\hline$\geq 20 \mathrm{~mm}$ & $8(66.7 \%)$ & $6(46.2 \%)$ & \\
\hline Growth pattern & & & 0.342 \\
\hline Endophytic & $11(91.7 \%)$ & $11(73.3 \%)$ & \\
\hline Exophytic & $1(8.3 \%)$ & $4(26.7 \%)$ & \\
\hline Mucosal layer ulceration & & & 0.569 \\
\hline Absent & $10(83.3 \%)$ & $14(93.3 \%)$ & \\
\hline Present & $2(16.7 \%)$ & $1(6.7 \%)$ & \\
\hline Schindler's sign & & & 0.075 \\
\hline Absent & $9(75.0 \%)$ & $15(100 \%)$ & \\
\hline Present & $3(25.0 \%)$ & 0 & \\
\hline Lesion consistence & & & 0.444 \\
\hline Firm & 11 (91.7\%) & $15(100.0 \%)$ & \\
\hline Soft & $1(8.3 \%)$ & 0 & \\
\hline
\end{tabular}

GISTs, gastrointestinal stromal tumors.

The color of the overlaying mucosa did not differ from the color of the surrounding mucosa in all GISTs and leiomyomas. In the majority of cases there were no ulcerations of the covering mucosa. There was a very prominent endophytic endoluminal grown pattern in $91.7 \%$ of GISTs and $73.3 \%$ of leiomyomas. Schindler's sign (convergence of the folds towards the lesion) was positive in $25 \%$ of GIST cases (Table 3 ).

More frequently GISTs were round in shape in comparison with leiomyomas. Heterogeneity of the SEL was observed more frequently in GISTs than in leiomyomas $(p=0.022)$. A hypoechogenic hyperechogenic image was more frequently seen in GISTs, whereas a hypoechogenic hypoechogenic image was more frequently observed in leiomyomas $(p=0.008)$. Marginal halo was observed in $25 \%$ of GISTs with no statistically significant difference comparing them to leiomyomas (Table 4).

Receiver operating characteristic curves were created to assess the discriminative value of tumor shape, heterogeneity, echogenicity, and tumor location other than in esophagus for predicting GISTs (Fig. 5).

Echogenicity $(\mathrm{AUC}=0.82[0.66-0.98])$ and tumor shape $(\mathrm{AUC}=0.81[0.64-0.97])$ demonstrated good pre-
Table 4. EUS features of patients with GISTs and leiomyomas

\begin{tabular}{|c|c|c|c|}
\hline Variables & GISTs & Leiomyomas & $p$ value \\
\hline Mean lesion size, $\mathrm{mm}$ & $25.25 \pm 11.41$ & $22.00 \pm 12.35$ & 0.489 \\
\hline EUS size groups & & & 0.651 \\
\hline$<10 \mathrm{~mm}$ & $1(8.3 \%)$ & $3(20.0 \%)$ & \\
\hline $10-19 \mathrm{~mm}$ & $3(25.0 \%)$ & $5(33.3 \%)$ & \\
\hline$\geq 20 \mathrm{~mm}$ & $8(66.7 \%)$ & $7(46.7 \%)$ & \\
\hline Lesion shape & & & 0.016 \\
\hline Round & $5(41.7 \%)$ & 0 & \\
\hline Oval & $7(58.3 \%)$ & $10(66.7 \%)$ & \\
\hline Circular & 0 & $4(26.7 \%)$ & \\
\hline Other & 0 & $1(6.7 \%)$ & \\
\hline Layer & & & 0.369 \\
\hline II & 0 & $2(13.3 \%)$ & \\
\hline III & $3(25.0 \%)$ & $1(6.7 \%)$ & \\
\hline IV & $9(75.0 \%)$ & $11(73.3 \%)$ & \\
\hline Unknown & 0 & $1(6.7 \%)$ & \\
\hline Lesion borders & & & 0.662 \\
\hline Regular & $8(66.7 \%)$ & $12(80.0 \%)$ & \\
\hline Irregular & $4(33.3 \%)$ & $3(20.0 \%)$ & \\
\hline Heterogeneity & & & 0.022 \\
\hline Heterogeneous & $8(66.7 \%)$ & $3(20.0 \%)$ & \\
\hline Homogenous & $4(33.3 \%)$ & $12(80.0 \%)$ & \\
\hline Echogenicity & & & 0.008 \\
\hline Hypoechogenic "hyper" & $6(50.0 \%)$ & $1(6.7 \%)$ & \\
\hline Hypoechogenic "iso" & $6(50.0 \%)$ & $8(53.3 \%)$ & \\
\hline Hypoechogenic "hypo" & 0 & $6(40.0 \%)$ & \\
\hline Marginal halo & & & 0.075 \\
\hline Absent & $9(75.0 \%)$ & 15 (100.0\%) & \\
\hline Present & $3(25.0 \%)$ & 0 & \\
\hline Cystic changes & & & 0.188 \\
\hline Absent & $10(83.3 \%)$ & 15 (100.0\%) & \\
\hline Present & $2(16.7 \%)$ & 0 & \\
\hline Calcification & & & 0.683 \\
\hline Absent & $9(75.0 \%)$ & $9(60.0 \%)$ & \\
\hline Present & $3(25.0 \%)$ & $6(40.0)$ & \\
\hline
\end{tabular}

EUS, endoscopic ultrasound; GISTs, gastrointestinal stromal tumors; "hyper", hyperechogenic; "hypo", hypoechogenic; "iso", isoechogenic.

dictive features. Heterogeneous EUS image (AUC $=0.73$ [0.53-0.93]) and tumor location other than esophagus (AUC $=0.77[0.59-0.95])$ showed satisfactory predictive feature (Table 5).

We were not able to detect one or two EUS features specific for GISTs that would differentiate GISTs from leiomyomas. However, the complex of EUS features could be helpful in diagnosing GISTs and differentiating GISTs from leiomyomas. As mentioned in Materials and Methods, in order to standardize the diagnostic approach to GISTs and leiomyomas, we assigned scores for seven upper endoscopic and EUS features (Table 1).

The mean total score in case of GISTs was significantly higher than that in leiomyomas: $3.25 \pm 1.71$ and $0.53 \pm$ $0.83(p<0.0001)$, respectively. Increment by one score increased the odds ratio for GISTs by 5.87 times (95\% CI $1.63-21.11 ; p=0.007)$. 
Table 5. Abilities of tumor location and EUS features of predicting GISTs

\begin{tabular}{lccc}
\hline EUS feature & AUC & $95 \%$ CI & $p$ value \\
\hline Tumor shape & 0.806 & $0.642-0.969$ & 0.007 \\
Heterogeneity & 0.733 & $0.534-0.932$ & 0.040 \\
$\begin{array}{l}\text { Echogenicity } \\
\text { Tumor location other than }\end{array}$ & 0.817 & $0.658-0.975$ & 0.005 \\
$\quad$ esophagus & 0.767 & $0.585-0.948$ & 0.019 \\
\hline
\end{tabular}

AUC, area under the receiver operating characteristic curve; EUS, endoscopic ultrasound; GISTs, gastrointestinal stromal tumors.

The total score demonstrated very good discriminatory features of GISTs against leiomyomas with the AUC $=0.942$ (95\% CI $0.858-1.000)$. The cutoff value of 1.5 total score had $83.3 \%$ sensitivity and $93.3 \%$ specificity in diagnosing GISTs.

\section{Discussion}

SELs in the UGT is a rare condition. In most cases they are diagnosed randomly during upper endoscopy and are asymptomatic. If the lesion size is $<10 \mathrm{~mm}$, an endoscopic follow-up within 1 year can be considered. In case of growth or the lesion becoming $>10 \mathrm{~mm}$, EUS should be performed. After upper endoscopy and EUS it is possible to make a definitive, presumptive, or uncertain diagnosis of SELs. In case of at least two presumptive or uncertain diagnoses of SELs, we try to find answers to the following questions: What is the best management option for the patient at this point? When should we perform biopsy of the SEL? When should we perform treatment directly even without preoperative pathological verification? What is the need and the intention of the patient to have the lesion treated?

Several management algorithms and guidelines for SELs have been produced [9-16]. Recently, the European Society of Gastrointestinal Endoscopy (ESGE) clinical guidelines regarding the indications, results, and clinical impact of EUS-guided sampling in gastroenterology were published [17]. Bite-on-bite biopsy should be the first diagnostic procedure for SELs. If the results are nondiagnostic, EUS-guided biopsy could be applied in the following clinical situations: (1) asymptomatic hypoechogenic SEL $\geq 20 \mathrm{~mm}$ of the stomach or gastroesophageal junction if surveillance is being considered; (2) targeted therapy of suspected GISTs is being considered; (3) if there are suspicions of carcinoma, neuroendocrine tumor, lymphoma, or intramural metastasis. The ESGE does not recommend EUS-guided sampling of SELs in the following clinical situations: (1) symptoms making resection

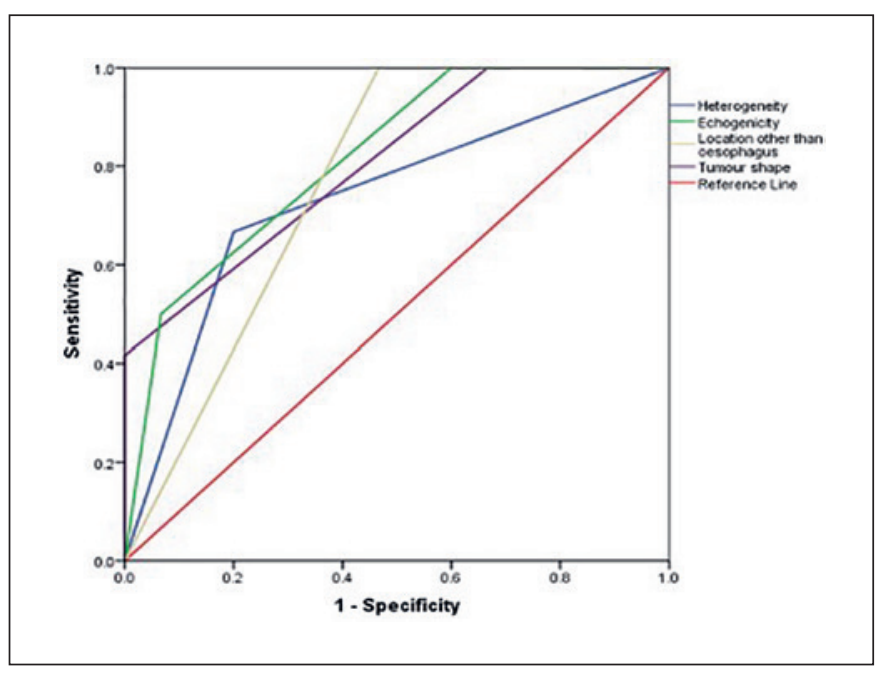

Fig. 5. Receiver operating characteristic curves of tumor location and endoscopic ultrasound features for predicting gastrointestinal stromal tumors.

necessary; (2) small ( $<20 \mathrm{~mm}$ ) SELs located in the esophagus or stomach; (3) pathognomonic EUS appearance of a lipoma or duplication cyst; (4) the patient is not a candidate for treatment. The European Society for Medical Oncology (ESMO) recommends that when the SELs in UGT are $<2 \mathrm{~cm}$ and the etiology is unknown, the standard approach will be EUS assessment followed by follow-up, reserving excision for patients whose tumor increases in size or becomes symptomatic. The standard approach to tumors $\geq 2 \mathrm{~cm}$ in size is biopsy/excision because they are associated with a higher risk of progression if confirmed as GISTs [16]. However, recommendations are weak with a low quality of evidence. There is no strong evidence to establish how often a follow-up examination should be performed when SELs are $<2 \mathrm{~cm}$. According to these guidelines and a practical standpoint, the critical size of SELs is $2 \mathrm{~cm}$. If the SEL is $\geq 2 \mathrm{~cm}$, the treatment could be performed even without preoperative biopsy. However, it seems that it is not enough to perform EUS only [7]. The results of this study revealed that in $42.9 \%$ of SELs $(>2 \mathrm{~cm})$ in which preoperative EUS was performed but without a histological examination after surgery, they appeared to be benign. It seems that preoperative biopsy of SELs should be performed. Overall, the diagnostic accuracy of EUS in diagnosing SELs using histopathology as a gold standard was $49,48,45.5$, and $66.7 \%$ [18-21]. The accuracy of EUS was $77.1 \%$ for GISTs, $50 \%$ for neuroendocrine tumor, $50 \%$ for ectopic pancreas, $100 \%$ for inflammatory fibroid polyps, and 25\% for leiomyomas [21]. What are the factors of inaccurate diagnosis of SELs after EUS examination? How could we increase EUS accuracy? In our study we aimed, in part, to approach these questions. This has also been done in other studies [5, 22-25]. 
Our study sample $(n=27)$ was specific in that we evaluated the upper endoscopic and EUS features of 12 GISTs and 15 leiomyomas with a mean size of $23.44 \pm$ $11.83 \mathrm{~mm}$ (range 7-50). According to our data, GISTs and leiomyomas were the main pathological diagnoses confirmed after deep biopsy via the endoscopic submucosal dissection technique [8]. We prospectively evaluated the upper endoscopic and EUS features of SELs before deep biopsy. Combining all these features, we made the presumptive diagnosis of SELs. In the GIST and leiomyoma groups the diagnosis was correct in $81.8 \%$ ( $p=$ 0.001 ) of cases, so we decided to find out the numerical value for these results with the aim of proposing a scoring system with a cutoff value to predict the probability of GIST. Retrospectively we created the scoring table with seven upper endoscopic and EUS parameters (Table 1). Four SELs features - localization, shape, heterogeneity, and echogenicity - were significantly different between the GIST and leiomyoma groups. No GIST was diagnosed in the esophagus and the stomach cardia. GISTs were more frequently round in shape, heterogenic, and hypoechogenic hyperechogenic compared to leiomyomas. A hypoechogenic hypoechogenic image was more frequently observed in leiomyomas, while a hypoechogenic isoechogenic image was equally observed in both lesions. Another three features - Schindler's sign, EUS "halo" sign, and EUS anechoic spaces - were observed only in GISTs. Nevertheless, these three features were not statistically significant $(p=0.075, p=0.075$, and $p=0.188)$ and the number of cases was small $(n=3, n=3$, and $n=2)$. It was controversial to choose which type of features as being accurate in making a scoring system and which score value for each feature could be optimal (Table 1). The mean total score in the GIST group was significantly higher than that in the leiomyoma group: $3.25 \pm 1.71$ and $0.53 \pm 0.83(p<0.0001)$, respectively. Increment by one score increased the odds ratio for GISTs by 5.87 times (95\% CI 1.63-21.11; $p=0.007)$. The total score demonstrated very good discriminatory features of GISTs against leiomyomas with an AUC of 0.942 (95\% CI 0.858-1.000). The cutoff value of 1.5 total score had $83.3 \%$ sensitivity and $93.3 \%$ specificity in diagnosing GISTs.

There are some limitations to the present study. It was a retrospective study and needs larger sample size. The scoring was evaluated only for GISTs and leiomyomas. How could we deal with other types of hypoechogenic SELs? The echogenicity of the SELs was not measured. The evaluation of this feature was only visual and was performed only by one investigator. The risk of interobserver disagreement is potential. In case of SELs with hypoechogenic isoechogenic image, it is difficult to differentiate GISTs from leiomyomas because in both groups this feature was almost equally observed. It could influence the accuracy of our diagnostic approach towards scoring the prediction of GISTs with false-positive or negative results. However, this drawback was resolved combining the complex of EUS and upper endoscopic features in diagnosing GISTs and differentiating them from leiomyomas. Our study analyzed SELs with a mean size of $23.44 \pm 11.83 \mathrm{~mm}$, and most of them had a regular shape. For larger-sized lesions with irregular margins and/or other EUS features, the scoring should be modified because size by itself is a very strong predictive feature.

Overall, not all questions related to the management of SELs are answered. Why do we need to improve EUS accuracy? How could it influence advanced EUS imaging, e.g., contrast-enhanced harmonic EUS, EUS-guided elastography, and EUS-guided needle-based confocal laser endomicroscopy? On the other hand, with regard to the strategy how to treat all hypoechogenic SELs $\geq 1 \mathrm{~cm}$ with a diagnostic/therapeutic intention, there is no need of such EUS imaging improvement. Nevertheless, there is no high-quality evidence concerning the management of SELs. The methods are debatable and the risk-benefit ratio and the cost-effectiveness of the approach towards these lesions need to be considered. GISTs are the most common mesenchymal neoplasms in the gastrointestinal tract and are mainly prevalent in the stomach. Our aim was to predict the probability of GISTs after upper endoscopic and EUS evaluations. In case of a high probability of GISTs $\geq 2 \mathrm{~cm}$, we could more accurately recommend the treatment even without a preoperative biopsy. In case of a high probability of GISTs $1-2 \mathrm{~cm}$ in size, we could suggest a better follow-up schedule. However, as an option, the patient could choose to undergo histological assessment with a diagnostic/therapeutic intention. In uncertain cases of SELs after EUS, biopsy of the lesions proves reasonable, particularly if their size is $>2 \mathrm{~cm}$.

Finally, we have created a recommended scoring system which needs to be discussed, corrected, and validated after future prospective cohort studies in an attempt to optimize the management of SELs.

\section{Conclusions}

Upper gastrointestinal endoscopy and EUS are the cornerstone methods in the management of SELs. Their diagnostic accuracy for a differential diagnosis of GISTs and leiomyomas is sufficient. In order to standardize the diagnostic approach to GISTs and leiomyomas, scores for seven upper endoscopic and EUS features were assigned. A cutoff value of 1.5 total score revealed $83.3 \%$ sensitivity and $93.3 \%$ specificity in diagnosing GISTs. In view of advanced EUS imaging techniques, SEL size, and different types of SELs, our proposed scoring system should be modified and evaluated in future prospective studies in order to enhance the management of SELs. 


\section{Statement of Ethics}

The study protocol was approved by the Vilnius Regional Research Ethics Committee within the Lithuanian Bioethics Committee and a written consent was obtained from all patients.

\section{Disclosure Statement}

The authors declare no conflict of interest.

\section{References}

1 Hedenbro JL, Ekelund M, Wetterberg P. Endoscopic diagnosis of submucosal gastric lesions. The results after routine endoscopy. Surg Endosc. 1991;5(1):20-3.

2 Stanaitis J, Vaicekauskas R, Lipnickas V, Valantinas J, Strupas K. Significance of Interdisciplinary Cooperation in the Treatment of Upper Gastrointestinal Mucosal and Submucosal Lesions: A Single Centre Experience. Visc Med. 2012;28(6):425-30.

3 Miettinen M, Sobin LH, Lasota J. Gastrointestinal stromal tumors of the stomach: a clinicopathologic, immunohistochemical, and molecular genetic study of 1765 cases with long-term follow-up. Am J Surg Pathol. 2005 Jan;29(1):52-68.

4 Kawanowa K, Sakuma Y, Sakurai S, Hishima T, Iwasaki Y, Saito K, et al. High incidence of microscopic gastrointestinal stromal tumors in the stomach. Hum Pathol. 2006 Dec; 37(12):1527-35.

5 Seo SW, Hong SJ, Han JP, Choi MH, Song JY, Kim HK, et al. Accuracy of a scoring system for the differential diagnosis of common gastric subepithelial tumors based on endoscopic ultrasonography. J Dig Dis. 2013 Dec;14(12): 647-53.

6 Codipilly DC, Fang H, Alexander JA, Katzka DA, Ravi K. Subepithelial esophageal tumors: a single-center review of resected and surveilled lesions. Gastrointest Endosc. 2018 Feb; 87(2):370-7.

7 Tae HJ, Lee HL, Lee KN, Jun DW, Lee OY, Han DS, et al. Deep biopsy via endoscopic submucosal dissection in upper gastrointestinal subepithelial tumors: a prospective study. Endoscopy. 2014 Oct;46(10):845-50.

8 Vaicekauskas R, Stanaitis J, Valantinas J. Efficacy of deep biopsy for subepithelial lesions in the upper gastrointestinal tract. Wideochir Inne Tech Malo Inwazyjne. 2016;11(3):192-
9 Eckardt AJ, Wassef W. Diagnosis of subepithelial tumors in the GI tract. Endoscopy, EUS, and histology: bronze, silver, and gold standard? Gastrointest Endosc. 2005 Aug; 62(2):209-12.

10 Alkhatib AA, Faigel DO. Endoscopic ultrasonography-guided diagnosis of subepithelial tumors. Gastrointest Endosc Clin N Am. 2012 Apr;22(2):187-205, vii.

11 Nishida T, Kawai N, Yamaguchi S, Nishida Y. Submucosal tumors: comprehensive guide for the diagnosis and therapy of gastrointestinal submucosal tumors. Dig Endosc. 2013 Sep;25(5):479-89.

12 Eckardt AJ, Jenssen C. Current endoscopic ultrasound-guided approach to incidental subepithelial lesions: optimal or optional? Ann Gastroenterol. 2015 Apr-Jun;28(2):16072.

13 Nishida T, Blay JY, Hirota S, Kitagawa Y, Kang YK. The standard diagnosis, treatment, and follow-up of gastrointestinal stromal tumors based on guidelines. Gastric Cancer. 2016 Jan;19(1):3-14

14 Hawes RH, Fockens P, Varadarajulu S, editors. Endosonography. Philadelphia: Elsevier Saunders; 2015.

15 Faulx AL, Kothari S, Acosta RD, Agrawal D, Bruining DH, Chandrasekhara V, et al.; Standards of Practice Committee. The role of endoscopy in subepithelial lesions of the GI tract. Gastrointest Endosc. 2017 Jun;85(6): 1117-32.

16 Casali PG, Abecassis N, Aro HT, Bauer S, Biagini R, Bielack S, et al.; ESMO Guidelines Committee and EURACAN. Gastrointestinal stromal tumours: ESMO-EURACAN Clinical Practice Guidelines for diagnosis, treatment and follow-up. Ann Oncol. 2018 Oct;29 Suppl 4:iv267.

17 Dumonceau JM, Deprez PH, Jenssen C, Iglesias-Garcia J, Larghi A, Vanbiervliet G, et al. Indications, results, and clinical impact of endoscopic ultrasound (EUS)-guided sampling in gastroenterology: European Society of Gastrointestinal Endoscopy (ESGE) Clinical Guideline - Updated January 2017. Endoscopy. 2017 Jul;49(7):695-714.
18 Reddymasu SC, Oropeza-Vail M, Pakseresht K, Moloney B, Esfandyari T, Grisolano S, et al. Are endoscopic ultrasonography imaging characteristics reliable for the diagnosis of small upper gastrointestinal subepithelial lesions? J Clin Gastroenterol. 2012 Jan;46(1): $42-5$.

19 Hwang JH, Saunders MD, Rulyak SJ, Shaw S, Nietsch H, Kimmey MB. A prospective study comparing endoscopy and EUS in the evaluation of GI subepithelial masses. Gastrointest Endosc. 2005 Aug;62(2):202-8.

20 Karaca C, Turner BG, Cizginer S, Forcione D, Brugge W. Accuracy of EUS in the evaluation of small gastric subepithelial lesions. Gastrointest Endosc. 2010 Apr;71(4):722-7.

21 Lim TW, Choi CW, Kang DH, Kim HW, Park SB, Kim SJ. Endoscopic ultrasound without tissue acquisition has poor accuracy for diagnosing gastric subepithelial tumors. Medicine (Baltimore). 2016 Nov;95(44):e5246.

22 Kim GH, Park DY, Kim S, Kim DH, Kim DH, Choi CW, et al. Is it possible to differentiate gastric GISTs from gastric leiomyomas by EUS? World J Gastroenterol. 2009 Jul;15(27): 3376-81.

23 Okai T, Minamoto T, Ohtsubo K, Minato H, Kurumaya H, Oda Y, et al. Endosonographic evaluation of c-kit-positive gastrointestinal stromal tumor. Abdom Imaging. 2003 MayJun;28(3):301-7.

24 Schulz RT, Fabio LC, Franco MC, Siqueira SA, Sakai P, Maluf-Filho F. Predictive features for histology of gastric subepithelial lesions. Arq Gastroenterol. 2017 Jan-Mar;54(1):115.

25 Kim GH, Kim KB, Lee SH, Jeon HK, Park DY, Jeon TY, et al. Digital image analysis of endoscopic ultrasonography is helpful in diagnosing gastric mesenchymal tumors. BMC Gastroenterol. 2014 Jan;14(1):7. 